\title{
Review on Optical Fiber Sensors With Sensitive Thin Films
}

\author{
Minghong YANG and Jixiang DAI
}

National Engineering Laboratory for Optical Fiber Sensing Technologies, Wuhan University of Technology, Wuhan, 430070, China

*Corresponding author: Minghong YANGＩE-mail: minghong.yang@whut.edu.cn

\begin{abstract}
The combination of fiber optics with nano-structure technologies and sensitive thin films offers great potential for the realization of novel sensor concepts. Miniatured optical fiber sensors with thin films as sensitive elements could open new fields for optical fiber sensor applications. Thin films work as sensitive elements and transducer to get response and feedback from environments, in which optical fibers are employed to work as signal carrier. This article presents some research work conducted at the National Engineering Laboratory for Optical Fiber Sensing Technologies in recent years. Concrete examples are: $\mathrm{Pd} / \mathrm{WO}_{3}$ co-sputtered coating as sensing material for optical hydrogen sensors shows robust mechanical stability and meanwhile good sensing performance; TbDyFe magnetostrictive coating directly deposited on fiber Bragg grating (FBG) demonstrates its possibility of miniature optical magnetic field/current sensors, and 40-pm shift of the FBG wavelength happens at a magnetic field order of $50 \mathrm{mT}$.
\end{abstract}

Keywords: Fiber Bragg grating, sensitive thin film, magnetron sputtering

\section{Introduction}

Optical fiber hydrogen sensor has been a research issue due to its excellent characteristics such as nature safety, small volume, and anti-electromagnetic interference. At present there are several kinds of optical fiber hydrogen sensors, such as evanescent sensor [1-6], micro-mirror sensor [7-8], surface plasmon resonance (SPR) sensor [9], acoustic resonator sensor [10], and fiber Bragg grating (FBG) sensor [11-14]. Although the evanescent and micro-mirror sensors are simple and inexpensive, their multiplexing capability is quite limited. Another disadvantage for the evanescent and micro-mirror sensors is that the sensor's accuracy is susceptible to the impact of the light source's intensity. Compared to SPR and acoustic resonator sensors, FBG is more suitable for real-time and distributed measurement. FBG sensor has been widely used in many industry applications due to its anti-electromagnetic interference, excellent distributed sensing possibilities, and ease of temperature compensation [15-17].

At present the most sensitive FBG hydrogen sensor is based on $\mathrm{WO}_{3}$ doped with $\mathrm{Pt}$ undergoing an exothermic reaction in hydrogen atmosphere [13]. The sensor features fast response and high sensitivity, but it still has the potential to explode in high concentrations of hydrogen. Sutapun and Tabib-Azar [12] reported that FBG coated with $560-\mathrm{nm} \mathrm{Pd}$ showed a linear sensitivity to $0.3 \% \mathrm{H}_{2}$ $-1.8 \% \mathrm{H}_{2}$, but the purity $\mathrm{Pd}$ film was so thick that the sensor's hydrogen response was irreversible, and FBG coated with thicker Pd film would have longer recovery time. It was also reported that reducing the diameter of the FBG could enhance the sensor's sensitivity in their research. M. Alexandra [14] demonstrated etched FBG sputtered with Pd thin

Received: 30 August 2011 / Revised version: 21 October 2011

(C) The Author(s) 2011. This article is published with open access at Springerlink.com 
film had higher sensitivity under different concentrations of hydrogen, but etched FBG was so fragile that it was not suitable for practical application. Side-polished FBG has an interesting structure that can increase sensitivity of the FBG sensors. Most researches focus on side-polished FBG's main characteristic which is sensitive to external refractive index, but its high bending sensitivity is rarely studied. J. Zhou [18] proposed that D-shaped FBG presented an intrinsic sensitivity to curvature, a characteristic that did not happen with the common FBG. It has been demonstrated that side-polished FBG (SP-FBG) coated with 20-nm Pd film showed that it was sensitive to hydrogen [19], but pure Pd films had poor adhesion to FBG due to their different physical properties. It is found that $\mathrm{WO}_{3} / \mathrm{Pd}$ composite film has good mechanical property [6]. To get good mechanical property and high sensitivity thin film, $\mathrm{WO}_{3}-\mathrm{Pd}$ composite film were deposited on SP-FBG to overcome the mismatch of material properties of the hydrogen sensitive film and $\mathrm{SiO}_{2}$. One shortcoming of the FBG coated with thin Pd film is that this kind of FBG is easily suffered from pure Pd film's fatal fracture caused by its $\alpha-\beta$ phase transition. Moreover pure $\mathrm{Pd}$ film has a poor adhesion to optic fiber, which will have a bad effect on the sensor's stability. To overcome these drawbacks, SP-FBG sputtered with $\mathrm{Pd} / \mathrm{Ag}$ composite film is developed in our lab. $\mathrm{Pd} / \mathrm{Ag}$ composite film has certain plasticity to tense strength during absorbing hydrogen, so it has been widely used in hydrogen separating industry. $\mathrm{Pd} / \mathrm{Ag}$ composite film is chosen as hydrogen sensitive film to overcome hysteretic effect during the absorbing and releasing hydrogen process. With the aim of enhancing the sensor's stability, Ti film is used as a basal layer to improve the adhesion between $\mathrm{Pd} / \mathrm{Ag}$ composite film and FBG. By side-polishing FBG to certain content, the sensor's sensitivity can be significantly improved.

In order to prepare hydrogen sensor with good performance, novel hydrogen sensor combined with SP-FBG and $\mathrm{Pd} / \mathrm{Ag}$ composite films, $\mathrm{WO}_{3}-\mathrm{Pd}$ composite films are proposed. $\mathrm{Pd} / \mathrm{Ag}$ and $\mathrm{WO}_{3}-\mathrm{Pd}$ composite films with different thicknesses are deposited on SP-FBG by magnetron sputtering technology, and SP-FBG hydrogen sensor coated with composite $\mathrm{Pd} / \mathrm{Ag}$ and $\mathrm{WO}_{3}-\mathrm{Pd}$ thin film as sensing media have been developed and its sensing characteristics have been investigated.

\section{Optical fiber hydrogen sensors with Pd-based composite films as sensitive materials}

$\mathrm{WO}_{3}-\mathrm{Pd}$ composite film consists of $\mathrm{WO}_{3}$, $\mathrm{WO}_{3} / \mathrm{Pd}$ composite film and $\mathrm{Pd}$ film. $\mathrm{WO}_{3}$ is deposited as basal layer because it has good adhesion to fiber. And Pd film is used as hydrogen sensitive film due to its high sensitivity and selectivity to hydrogen. $\mathrm{WO}_{3} / \mathrm{Pd}$ composite film is set as an intermediate layer to overcome material properties' mismatch of $\mathrm{WO}_{3}$ and $\mathrm{Pd}$. The other reason for adding $\mathrm{WO}_{3} / \mathrm{Pd}$ composite film is to improve the $\mathrm{WO}_{3}-\mathrm{Pd}$ film's mechanical property. When the thickness of the pure Pd thin film is more than $40 \mathrm{~nm}$, it is so easy to crack because of the accumulation of lattice dislocations caused by its volume expansion. By adding the intermediate layer, the accumulation of lattice dislocations of single $\mathrm{Pd}$ layer can be reduced. So the repeatability of $\mathrm{WO}_{3}-\mathrm{Pd}$ composite film will be enhanced. The FBG sensing head with $40-\mathrm{nm} \mathrm{WO}_{3}-\mathrm{Pd}$ composite film is firstly prepared by a $5-\mathrm{nm} \mathrm{WO}_{3}$ coating realized with radio frequency (RF) sputtering process, followed by depositing 5-nm $\mathrm{WO}_{3} / \mathrm{Pd}$ composite film, and then finished with $30-\mathrm{nm} \mathrm{Pd}$ film by direct-current (DC) sputtering method. Meanwhile, FBG and SP-FBG coated with $110-\mathrm{nm}^{\mathrm{WO}_{3}}-\mathrm{Pd}$ composite film are prepared by alternately adding $5-\mathrm{nm} \mathrm{WO}_{3} / \mathrm{Pd}$ composite film and 30-nm Pd film two times for comparison.

A Lambda Physik excimer laser (COMPex-150T) operating at $248 \mathrm{~nm}$ was used as laser source to write FBG in SMF-28 by the phase mask method [20-21]. The optical cladding of the FBG fiber was 
mechanically ground by a motor-driven polishing wheel [22] and polished to about several micro-meters of minimal residual thickness near the fiber core. During the side-polishing process, the FBG fiber was connected to light source and optic power meter to avoid polishing excessively. By controlling side-polishing time and the optical power loss, FBG was polished to different contents during our experiment. After the side-polishing process, the widths of side face of SP-FBGs were measured by VHX-100 digital microscope. As shown in Fig. 1, the widths of SP-FBG-1, SP-FBG-2, SP-FBG-3 and SP-FBG-4 are $124.7 \mu \mathrm{m}, 124.8 \mu \mathrm{m}$, $124.5 \mu \mathrm{m}, 124.3 \mu \mathrm{m}$, respectively. The SP-FBG-2 has the deepest side-polishing content because it has the largest width.

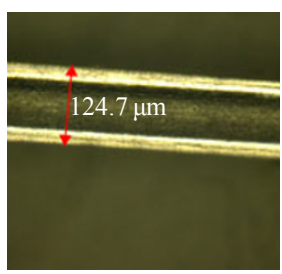

(a)

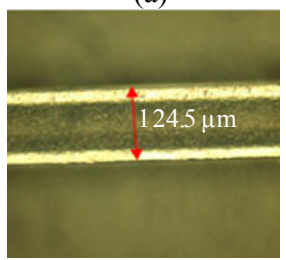

(c)

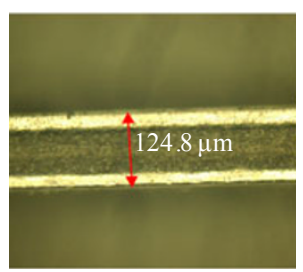

(b)

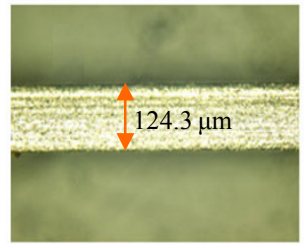

(d)
Fig. 1 Microphotography of side-polished FBG: (a) SP-FBG-1 top view after side-polished, (b) SP-FBG-2 top view after side-polished, (c) SP-FBG-3 top view after side-polished, and (d) SP-FBG top view after composite coating.

$\mathrm{WO}_{3}-\mathrm{Pd}$ and $\mathrm{Pd} / \mathrm{Ag}$ composite thin films are coated on the side-polished FBG fiber by using a BESTECH sputtering system. The system is equipped with DC and RF sputtering sources. 3-inch $\mathrm{Pd}$ and $\mathrm{WO}_{3}$ targets are installed to $\mathrm{DC}$ and $\mathrm{RF}$ sources, and the distance between the FBG and substrate-target is about $150 \mathrm{~mm}$. Meanwhile, several $10 \mathrm{~mm} \times 10 \mathrm{~mm} \mathrm{Si}$ pieces are set in the chamber for further characterization. Under $0.5-\mathrm{Pa}$ sputtering pressure of Ar, the deposition power for $\mathrm{Pd}$ and $\mathrm{WO}_{3}$ targets are $100 \mathrm{~W}$ and $150 \mathrm{~W}$ respectively, which correspond to deposition rate of $1.4 \mathrm{~nm} / \mathrm{s}$ and $0.4 \mathrm{~nm} / \mathrm{s}$, respectively. $\mathrm{Pd} / \mathrm{Ag}$ composite thin films are deposited on the side-polished FBG fiber by using a BESTECH sputtering system. FBG sensing heads with $50-\mathrm{nm}$ and $110-\mathrm{nm} \mathrm{Pd} / \mathrm{Ag}$ composite films are then finished by depositing 50-nm and 110-nm $\mathrm{Pd} / \mathrm{Ag}$ composite films respectively with co-sputtering process. Under 0.5-Pa sputtering pressure of Ar, deposition power for $\mathrm{Pd}$ and $\mathrm{Ag}$ targets are $100 \mathrm{~W}$ and $55 \mathrm{~W}$ respectively, which correspond to deposition rate of $0.14 \mathrm{~nm} / \mathrm{s}$ and $0.05 \mathrm{~nm} / \mathrm{s}$, respectively. Under this sputtering process, the atomic ratio of $\mathrm{Pd}$ and $\mathrm{Ag}$ is approximately 76:24. During the sputtering process, the thickness of the $\mathrm{Pd} / \mathrm{Ag}$ composite film is monitored by quartz crystal method.

Figure 2 is the schematic diagram of the fiber-hydrogen sensor. The sensing FBG and temperature compensating FBG are connected to an SLED light with the maximum power of $85 \mu \mathrm{W}$ by a 3-dB coupler. The varying hydrogen concentrations are provided by changing flowing rate of $\mathrm{H}_{2}$ and $\mathrm{N}_{2}$. When different concentration hydrogen is input in the gas room, the hydrogen-sensitive materials' resistance will be changed in different degrees. During hydrogen concentration characterization, the reflected wavelength is collected with a BCD-100 FBG demodulator. The measured data is recorded by a computer connecting with the FBG demodulator by communication interface for further data treatment.

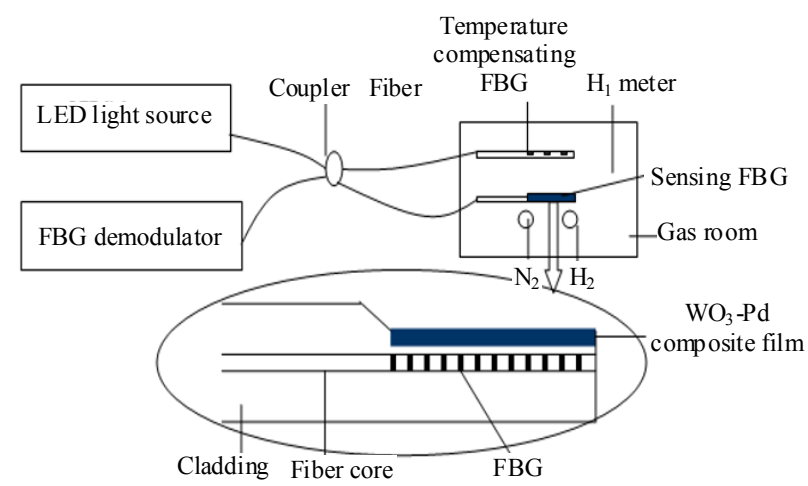

Fig. 2 Configuration of optical fiber hydrogen sensor characterization

Figure 3 illustrates the center wavelength shift of the FBG coated with $40-\mathrm{nm}$ and $110-\mathrm{nm} \mathrm{WO}_{3}-\mathrm{Pd}$ 
composite films under different concentration hydrogen. It can be seen that all FBGs coated with the hydrogen sensitive film shift to longer wavelength with an increase of the hydrogen's concentration. And the wavelength shift of the FBG has a nonlinear relationship with the hydrogen's concentration. When the hydrogen's concentration is $6 \%$ in volume percentage, $40-\mathrm{nm} \quad \mathrm{WO}_{3}-\mathrm{Pd}$ composite film saturates, and the wavelength shifts of FBG and SP-FBG-1 are $5 \mathrm{pm}$ and $15 \mathrm{pm}$, respectively. Common FBG, SP-FBG-2, SP-FBG-3 and SP-FBG-4 coated with $110-\mathrm{nm} \quad \mathrm{WO}_{3}-\mathrm{Pd}$ composite film saturate at $8 \%$ hydrogen, and corresponding wavelength shifts are $16 \mathrm{pm}, 40 \mathrm{pm}$, $45 \mathrm{pm}$ and $55 \mathrm{pm}$, respectively. Because of the different polishing depths of the fiber, SP-FBG-2, SP-FBG-3 and SP-FBG-4 have different sensitivities to hydrogen. FBG coated with thicker film has more wavelength shift, but the response rate will be sacrificed. The response time of the FBG coated with $40-\mathrm{nm} \mathrm{WO}_{3}-\mathrm{Pd}$ composite film is about $40 \mathrm{~s}$, and that for FBG sputtered with $110-\mathrm{nm} \mathrm{WO}_{3}-\mathrm{Pd}$ composite film is about $90 \mathrm{~s}$.

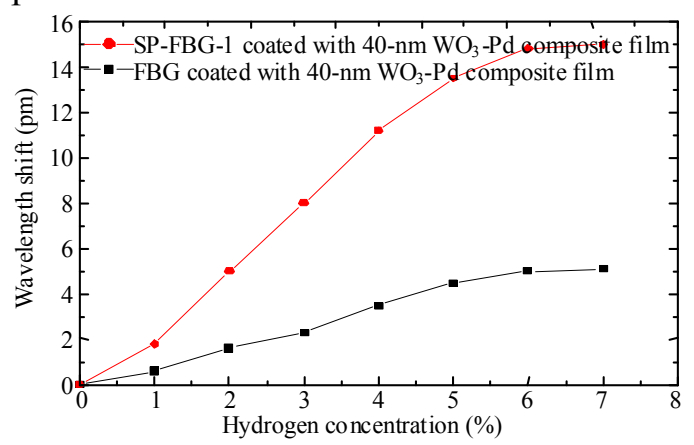

(a)

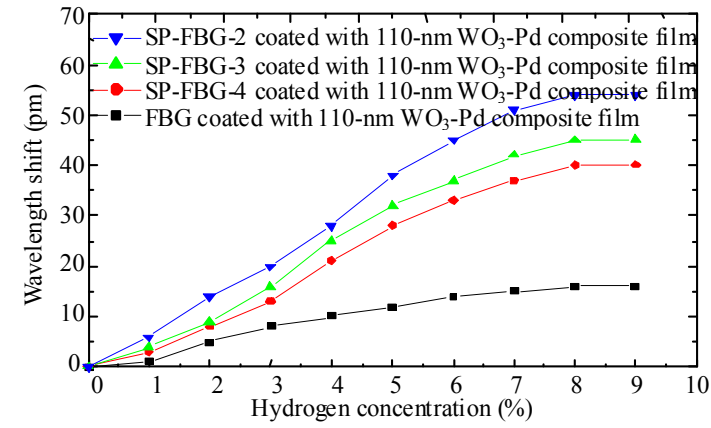

(b)

Fig. 3 Center wavelength shift of the common FBG and SP-FBG coated with 40-nm (a) and 110-nm (b) $\mathrm{WO}_{3}-\mathrm{Pd}$ composite films.
It is interesting to find that SP-FBG greatly increase sensitivity compared to common FBG coated with the same film. For $40-\mathrm{nm} \mathrm{WO}_{3}-\mathrm{Pd}$ composite film, SP-FBG's wavelength shift is three times as that of the common FBG exposed to the same concentration hydrogen. Compared to common FBG, the sensitivities of SP-FBG-2, SP-FBG-3 and SP-FBG-4 coated with 110-nm $\mathrm{WO}_{3}-\mathrm{Pd}$ composite film are increased by $244 \%$, $181 \%$ and $150 \%$, respectively. These results can be explained by the deduction of the effective cross-area and high bending sensitivity of the SP-FBG. It was reported [18] that the bending sensitivity of the D-shaped FBG was about 80 times higher than that of the normal circularly symmetrical FBG. When FBG was side-polished, it would form D-shaped cross section, and there was slight outward curvature on the fiber. This was why SP-FBG could increase the sensitivity of the sensor more than $100 \%$.

Owing to the highest sensitivity of SP-FBG-2, further investigation into the SP-FBG-2 was carried out in our experiment. Figure 4 displays the hydrogen response of SP-FBG-2 under different concentration hydrogen. From Fig. 4 we can get that the SP-FBG-2 has reversible response under different concentration hydrogen. When the hydrogen's volume ratio is $4 \%$, the center wavelength shift of SP-FBG-2 is about $25 \mathrm{pm}$. And SP-FBG-2 has an approximately 5-pm

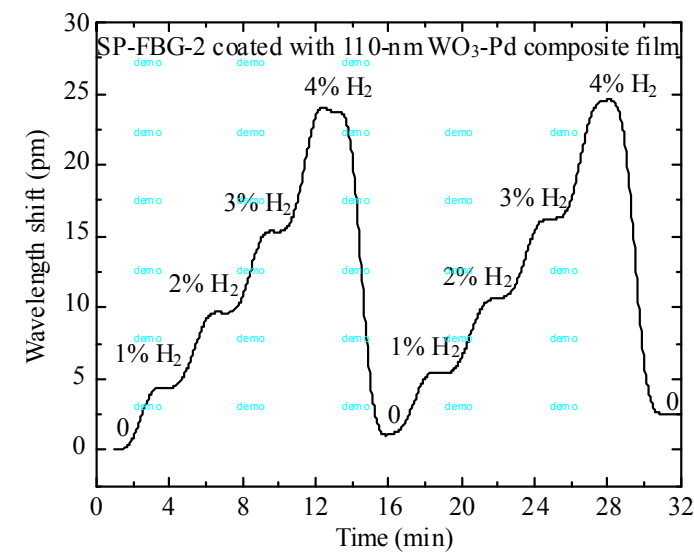

Fig. 4 Two cycles of SP-FBG-2 coated with $110-\mathrm{nm} \mathrm{WO}_{3}-$ Pd composite film under different concentrations hydrogen. 
response to $1 \%$ hydrogen. The center wavelength of SP-FBG-2 can not be restored to its initial value, and this could be attributed to the imperfect restoration of sensitive films. The hydrogen sensitive material's microstructure and the design of the sensor's structure will be investigated in future.

Figure 5 illustrates the central wavelength shifts of the FBG coated with $40-\mathrm{nm}$ and $110-\mathrm{nm} \mathrm{Pd} / \mathrm{Ag}$ composite films under different hydrogen concentrations. All central wavelengths of FBGs shift to longer with an increase of the hydrogen concentration, and the FBG wavelength shift has a nonlinear relationship with the hydrogen concentration. When the hydrogen concentration is $1 \%$, there is almost no wavelength shift for standard FBG coated with $50-\mathrm{nm} \mathrm{Pd} / \mathrm{Ag}$ composite film. It is difficult for hydrogen atomic to penetrate the hydrogen sensitive film if the hydrogen pressure is not high enough. By increasing the hydrogen concentration to $2 \%$, the wavelength shift of the FBG increases quickly. When the hydrogen concentration is $4 \%$ in volume percentage, the wavelength shifts of FBG and SP-FBG-1 are $4 \mathrm{pm}$ and $8 \mathrm{pm}$, respectively. For $110-\mathrm{nm} \mathrm{Pd} / \mathrm{Ag}$ composite film, the corresponding wavelength shifts are $8 \mathrm{pm}$ and $18 \mathrm{pm}$, respectively. Because of the different polishing depths of the fiber, SP-FBG-1 and SP-FBG-2 have different sensitivities to hydrogen. FBG coated with thicker film has more wavelength shift, but it has longer response time. The response time of the FBG coated with $50-\mathrm{nm}$ $\mathrm{Pd} / \mathrm{Ag}$ composite film is about $60 \mathrm{~s}$, and that for FBG sputtered with 110-nm $\mathrm{Pd} / \mathrm{Ag}$ composite film is about $140 \mathrm{~s}$. The recovery time for FBG with 40-nm and $110-\mathrm{nm} \mathrm{Pd} / \mathrm{Ag}$ composite films is $70 \mathrm{~s}$ and $150 \mathrm{~s}$, respectively. Here we define response time in the way that the time needed for maximum wavelength shift when exposed to different hydrogen concentrations, while omitting the transferring time of the hydrogen gas to sensors.

Compared to our previous work, the sensitivity of $\mathrm{Pd} / \mathrm{Ag}$ is less than that of the $\mathrm{WO}_{3}-\mathrm{Pd}$ composite film. The reason for this phenomenon is $\mathrm{Pd} / \mathrm{Ag}$ composite film has smaller volume expansion during hydrogen explosion. Ag is not sensitive to hydrogen, so $\alpha-\beta$ phase transition of the hydrogen sensitive film can be suppressed by alloying with Ag. The lattice constant difference between $\alpha$ and $\beta$ phases of the $\mathrm{Pd} / \mathrm{Ag}$ composite film is less than that of the pure Pd film, which can reduce lattice defect during $\alpha-\beta$ phase transition. So FBG coated with $\mathrm{Pd} / \mathrm{Ag}$ composite film has less sensitivity to hydrogen. However the hydrogen sensor's repeatability can be improved by using $\mathrm{Pd} / \mathrm{Ag}$ alloys.

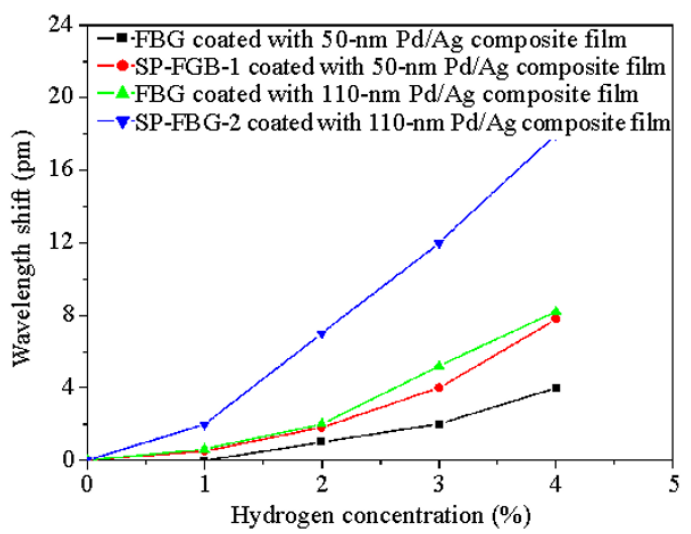

Fig. 5 Central wavelength shifts of the FBG and SP-FBG coated with $50-\mathrm{nm}$ and 110-nm $\mathrm{Pd} / \mathrm{Ag}$ composite films under different hydrogen concentrations.

Figure 6 shows the wavelength shifts of three cycle's hydrogen response of the FBG and SP-FBG coated with $50-\mathrm{nm}$ and $110-\mathrm{nm} \mathrm{Pd} / \mathrm{Ag}$ composite films. During the three cycle's response, the sensor has good repeatability to $4 \%$ hydrogen concentration. There is little hysteretic effect on the hydrogen response. Another reason may be that the mechanical strength of the $\mathrm{Pd} / \mathrm{Ag}$ composite film is higher than that of the pure $\mathrm{Pd}$ film, which is correspondence with the good appearance of the $\mathrm{Pd} / \mathrm{Ag}$ composite film in Fig. 4.

Compared to the standard FBG coated with the same film, SP-FBG can greatly increase the hydrogen sensor's sensitivity. The hydrogen sensitive film may have higher adhesion to the rough side-face of the SP-FBG. And SP-FBG has 
little effective cross section area. It was reported that the bending sensitivity of the D-shaped FBG was about 80 times higher than that of the standard FBG. When FBG is side-polished, it will form D-shaped cross section, and there is slight outward curvature on the fiber. This is why SP-FBG can increase the sensitivity of the sensor more than $100 \%$. Furthermore, increasing $\mathrm{Pd} / \mathrm{Ag}$ composite film's thickness can enhance the sensor's sensitivity. D-shaped FBG can also be used to improve the sensor's performance.

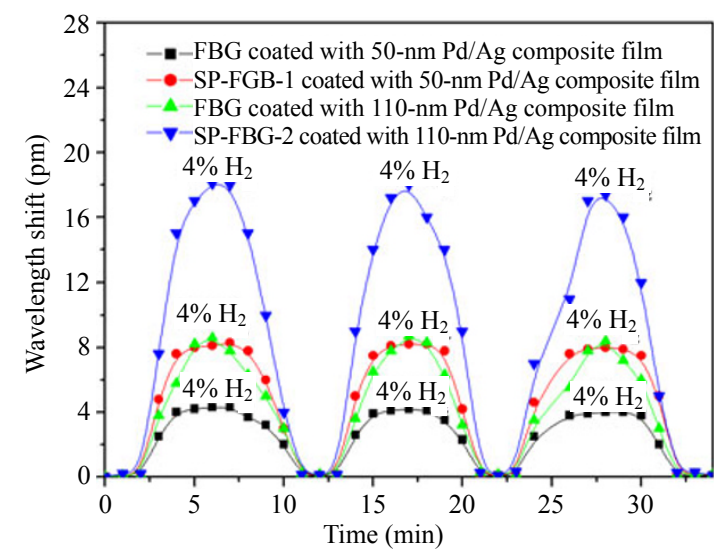

Fig. 6 Three cycle's hydrogen response of the standard FBG and SF-FBG coated with $50-\mathrm{nm}$ and $110-\mathrm{nm} \mathrm{Pd} / \mathrm{Ag}$ composite films.

\section{Optical fiber magnetic filed sensor development}

Great interest in giant magnetostrictive thin film has been grown over the past few years due to its potential as powerful transducer for the realization of microactuators. Meanwhile, they can be easily scaled down to micro- or nano-structure. Many efforts have been concentrated on the development of the magnetostrictive fiber sensor, in which strain in a magnetostrictive material resulted from an applied magnetic field is transferred to an optical fiber [23-25]. In principle, a fiber grating can detect magnetic field by Faraday effect, and this mechanism is ideal since no external transducer is required. However, its sensitivity is too low for practical application. Properties like electric or magnetic fields can be detected by fiber grating using magnetostirictive transducers [26]. Optical fiber magnetic/current field sensors have been proposed for decades. The measurement of small changes in optical path length can be accomplished using phase-modulating Mach-Zehnder interferometer [27]. It is demonstrated that a nickel coated optical fiber shows that it is sensitive to magnetic field [28]. Sensitivity can be further improved by using proper composition of metallic glasses. It has also been demonstrated that optical fiber jacketing with a magnetostrictive material is more efficient compared to simply gluing a fiber to a flat strip [29]. A disadvantage of using metallic glass jacket is that metallic and metallic glass materials exhibit saturation of magnetostriction at comparatively low magnetic fields, therefore this sensing element may be undesirably sensitive to small current field fluctuation [30]. A magnetostrive sensor using Terfenol-D and $\mathrm{Ni}_{65} \mathrm{Cu}_{33} \mathrm{Fe}_{2}$ epoxy-bonded with the FBG fiber is proposed for DC-current and temperature discrimination [31], however, this method is based on bulk magnetostrictive materials in size of $6 \mathrm{~mm} \times 25 \mathrm{~mm}$, which is problematic for miniature application.

Fiber optical sensors offer several significant advantages over conventional electrical sensors. They show no interference with electromagnetic radiation, so they can function in many hostile environments where conventional sensors would fail $[32,33]$. TbDyFe is one of the most promising magnetostrictive materials, especially $\mathrm{TbDyFe}$ has giant magnetostrictive coefficient [34, 35]. It will expand when being put in magnetic field, which has a linear and reverse response to the strength of the magnetic field. If these magnetostrictive materials are coated onto an FBG fiber, the elongation of the magnetostrictive film due to magnetic field will result in a change in grating period in FBG fiber, and therefore generate a shift of the FBG central wavelength. In this way the drift of the FBG wavelength to the magnetic field response is correlated. Using TbDyFe magnetostrictive material as sensing element has been proposed, however 
most of them use bulk TbDyFe glued to a fiber. To our knowledge, there is no report concerning single TbDyFe coating as sensing element. The associated problem is that magnetostrictive response relies on the size of materials used; the response with the thin film could be too weak for application compared to bulk materials. The improvement of the sensitivity would be a key issue for magnetostictive thin film materials. Exchanging magnetostriction in giant magnetostorictive hard-magnetic layer and soft-magnetic high magnetization layer was reported [14], and this could be also an effective way to improve magnetostrictive response and therefore to enhance magnetic field sensitivity.

We proposed an idea of depositing magnetostrictive TbDyFe thin films on cladding-etched FBGs. From the principle of mechanics, at the same stress, the strain is proportional to the mass to be affected; an improvement of the sensitivity by magentostrictive material in magnetic field with less mass of the fiber due to etching process could be anticipated. Therefore, in order to improve sensitivity, fiber grating sensors with the same TbDyFe layer thickness, but different cladding thickness after etching process are also prepared and characterized. Meanwhile, magenetostrictive multilayer is also investigated with the interest to improve the sensitivity of the magnetic field response.

Single mode FBG fiber was dipped into mixed hydrofluoric acid (HF) solvent to remove cladding layer. The HF solvent was diluted by propanone and de-ioned water with 1:1:10 in volume. The etching process was carried out at room temperature; fiber diameter was measured with optical microscopy after the etching process. An average etching speed of $20 \mu \mathrm{m} / \mathrm{h}$ was concluded. For preparing the fiber with different thicknesses, we stopped the process with different total etching time, and finally the etched fibers with outer cladding diameters of $85 \mu \mathrm{m}$, $105 \mu \mathrm{m}$ were prepared. The etched FBG length was about $15 \mathrm{~mm}$.
Thin films of the giant magnetostrictive material TbDyFe were coated on the etched single mode optical fiber. A BESTECH sputtering system was used to prepare these thin films. The system was specially designed for optical fiber coating and equipped with DC and RF sputtering sources. It had turbo pump and allowed a basic vacuum pressure down to $10^{-9}$ mbar. 3-inch TbDyFe targets were installed to RF sources, and the pre-treated optical fibers were located at a position with substrate-target distance of $150 \mathrm{~mm}$. Meanwhile, two $10 \mathrm{~mm} \times 10 \mathrm{~mm}$ $\mathrm{Si}$ pieces were used as monitoring sample in order to characterize the deposited layer later. Deposition power for TbDyFe targets was $150 \mathrm{~W}$, which responded to a deposition rate of about $0.08 \mathrm{~nm} / \mathrm{s}$. TbDyFe single layers with the same thickness of about $0.8 \mu \mathrm{m}$ were sputtered on fiber with different etched cladding diameters ( $85 \mu \mathrm{m}, 105 \mu \mathrm{m}$, and $125 \mu \mathrm{m})$ as described before. For comparison FeNi layer and $\mathrm{TbDyFe} / \mathrm{FeNi}$ multilayer were also deposited on non-etched FBG fibers.

The setup of the magnetic field sensing characterization system is schematically shown in Fig. 7. An SLED light with the maximum power of $85 \mu \mathrm{W}$ is coupled to two FBG fibers with one coated with $\mathrm{TbDyFe}$ single layer or $\mathrm{TbDyFe} / \mathrm{FeNi}$ multilayer. These two fibers are inserted into spiral coil in parallel as sensing element and compensating element. The diameter size of the spiral coil is $10 \mathrm{~mm}$, and totally there are 1000 turns as designed. A Lake Shore 460 Gauss meter is employed to calibrate the magnetic intensity as shown in Fig. 8. Magnetic intensity corresponds to $50 \mathrm{mT}$ when DC current rises up to $3000 \mathrm{~mA}$. The reflected wavelength is collected with a BCD-100 FBG demodulator in magnetic field characterization. This demodulator is equipped with a fiber Fabry-Perot tunable filter (FFP-TF) from Micron Optics Inc., USA as core component for wavelength demodulation. Compared to the usually-used optical spectrum analyzer based on scanning grating technology, the FFP-TF is a real fiber etalon, which 
preserves the advantage of the high optical resolution, while avoids extreme alignment, temperature, and vibration sensitivity existed in bulk optic Fabry-Perot interferometer. The resolution of this FFP-TP demodulator is $1 \mathrm{pm}$, while the usually-used ADVANTEST Q8347 can only provide spectrum resolution of $0.01 \mathrm{~nm}$. Meanwhile, a standard non-coated FBG fiber is used here as reference to compensate wavelength shift due to temperature drift and background noise. The measured data are recorded and sent to computer for further data processing.

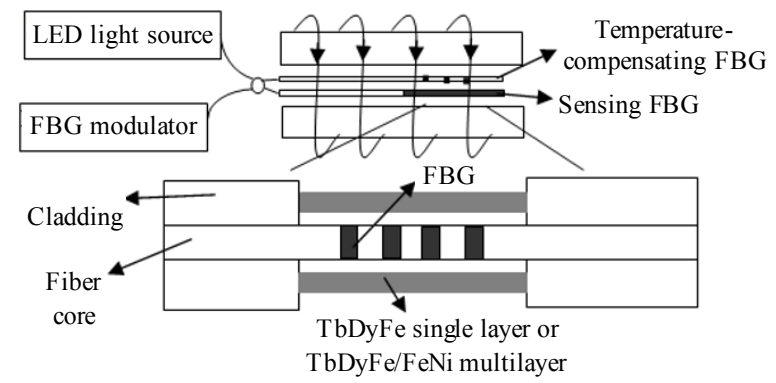

Fig. 7 Configuration of the optical fiber magnetic sensor characterization.

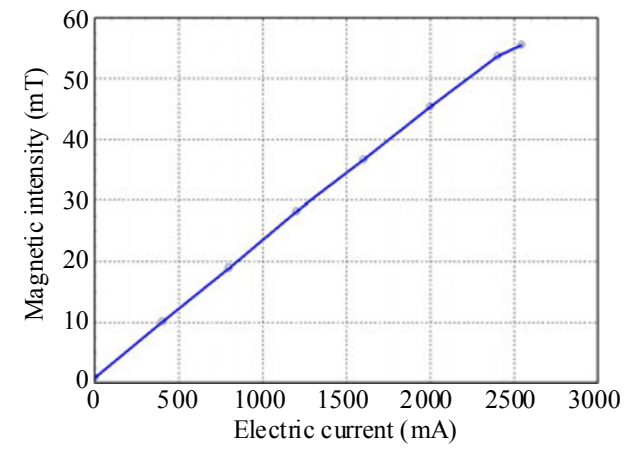

Fig. 8 Calibrated magnetic field intensity induced by spiral coil current with a Lake Shore 460 Gauss meter.

Figure 9 shows the drift of the FBG wavelength (cladding-etched) during an increase of the magnetic field up to $50 \mathrm{mT}$, since a pure FBG sensor is employed to compensate surrounding effect such as temperature change, and the drift of the measured FBG wavelength can be solely regarded as the effect of the magnetic field change due to different magnetic field levels. Therefore the correlation of the magnetic field and the shift of the FBG wavelength is maintained. It can be also concluded from Fig. 7 that cladding-etched fiber shows more sensitivity to magnetic field change. With the same $0.8-\mu \mathrm{m}$ TbDyFe coating, the sensitivity responses of the FBG wavelength shift for non-etched $(125 \mu \mathrm{m}$ in diameter), one-hour etched (105 $\mu \mathrm{m}$ in diameter) and 2-hour etched ( $85 \mu \mathrm{m}$ in diameter) are $0.386 \mathrm{pm} / \mathrm{mT}$, $0.563 \mathrm{pm} / \mathrm{mT}$ and $0.950 \mathrm{pm} / \mathrm{mT}$, respectively. These results prove the correctness of the supposition that the strain is proportional to the mass being affected, and an improvement of the sensitivity with less mass of the fiber due to etching process has been demonstrated. The more the etched cladding layer is, the stronger the response will be.

Since the magnetostrictive TbDyFe coatings on different FBGs are the same, their improvement of the sensitivity could be compared. When it is normalized with the non-etched FBG, it can be concluded that the sensitivity of 2-hour etched FBG is about 2.5 times higher than that of the standard non-etched FBG, while it is only 1.4 times higher in case of one-hour etched FBG. In this sense, it is better to etch the outer cladding layer as thin as possible in order to enhance sensitivity to magnetic field. However during thin film deposition, FBG fibers with such thin diameter is prone to break, therefore it is not practically feasible to etch FBG cladding layer too thin. Normally we etched FBG fibers to $85 \mu \mathrm{m}$ with a length of about $15 \mathrm{~mm}$.

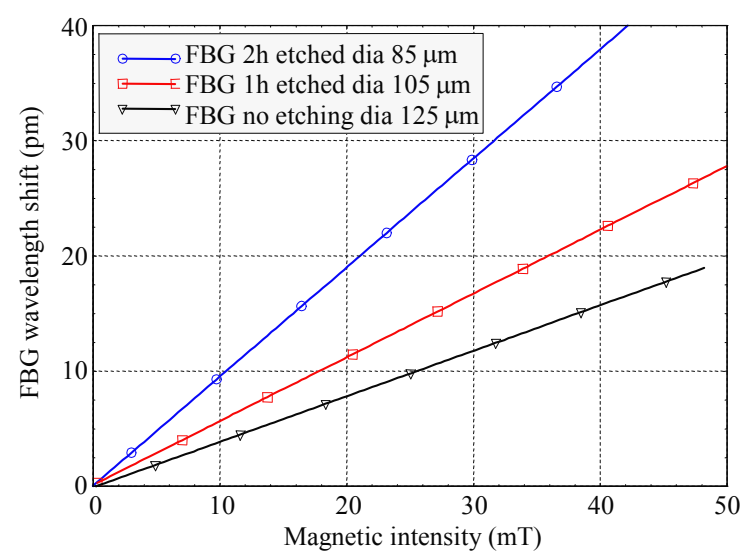

Fig. 9 Central wavelength shift of three FBGs deposited with the same $0.8-\mu \mathrm{m}$ TbDyFe coating, but with different chemically-etched cladding thicknesses. 
Similarly, 1- $\mu \mathrm{m}$ FeNi coating, 1- $\mu \mathrm{m}$ TbDyFe and $0.5-\mu \mathrm{m} \mathrm{FeNi} / 0.5-\mu \mathrm{m}$ TbDyFe multilayer are prepared on the standard $125-\mu \mathrm{m}$ single-mode FBGs. Their performances are characterized for comparison under the same condition as shown in Fig. 10. In the case of single layer, TbDyFe coating shows stronger response to the magnetic field than FeNi coating, which can be understood the the fact that the magnetostrictive coefficient of TbDyFe is greater than that of FeNi. Furthermore when compared with the former single TbDyFe coating $(0.8 \mu \mathrm{m}$ in thickness) as shown in Fig. 9 , a slight improvement of the sensitivity with thicker TbDyFe sensitive coating can be observed. The sensitivity response of the FBG wavelength shift is $0.523 \mathrm{pm} / \mathrm{mT}$ for the non-etched FBG with 1- $\mu \mathrm{m}$ TbDyFe coating, which is a little higher than the former one as mentioned in Fig. 9.

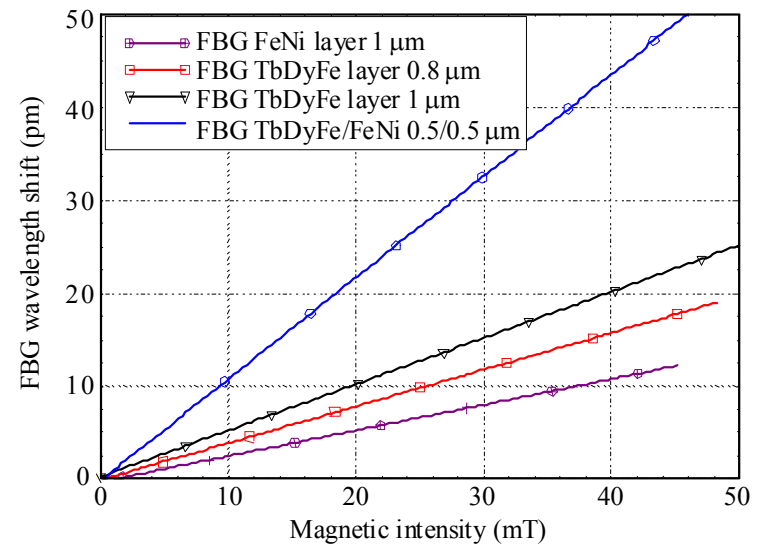

Fig. 10 Central wavelength shift of four standard single-mode FBGs (unetched) deposited with 1- $\mu \mathrm{m}$ FeNi, 0.8- $\mu \mathrm{m}$ TbDyFe, 1- $\mu \mathrm{m}$ TbDyFe, and 0.5- $\mu \mathrm{m}$ TbDyFe/0.5- $\mu \mathrm{m}$ FeNi, respectively.

However, when compared to multilayer, $0.5-\mu \mathrm{m}$ FeNi/0.5- $\mu \mathrm{m}$ TbDyFe multilayer shows the strongest sensitivity. The central wavelength shift of the FBG coated with the above-mentioned multilayer is nearly $45 \mathrm{pm}$ at the magnetic field level of $40 \mathrm{mT}$. This shift is only $20 \mathrm{pm}$ for $1-\mu \mathrm{m}$ TbDyFe single layer, and $11 \mathrm{pm}$ in the case of FeNi single layer. This has demonstrated the possibility to improve the magnetostrictive response and therefore to enhance the magnetic field sensitivity. However, this is not possible for the bulk TbDyFe material.

From further review on the experimental results, it can be concluded that the $\mathrm{TbDyFe} / \mathrm{FeNi}$ multilayer has sensitivity response of $1.08 \mathrm{pm} / \mathrm{mT}$, which is already two times higher than that of the $1-\mu \mathrm{m}$ TbDyFe single layer. Even compared to the best result achieved by side-etching of the FBG cladding layer, the sensitivity with the multilayer is still a bit higher. It can be expected that the response sensitivity can be further improved by depositing multilayer on the side-etched FBG.

It should be mentioned that the FBG wavelength shift is quite linear in the case of coating or structure designs. This attributes to the good performance of the TbDyFe giant magnetostrictive material, which has a linear and reverse response to the strength of the magnetic field. This linear response is very promising for sensor application.

Magnetic filed sensors based on magnetostrictive thin films have been proposed, and two kinds of methods to improve sensitivity have been demonstrated. Sputtered magnetostrictive TbDyFe coatings are deposited on different cladding-etched FBGs. The sensitivity response of the FBG wavelength shift for non-etched $(125 \mu \mathrm{m}$ in diameter), one-hour etched (105 $\mu \mathrm{m}$ in diameter) and 2-hour etched ( $85 \mu \mathrm{m}$ in diameter) are $0.386 \mathrm{pm} / \mathrm{mT}$, $0.563 \mathrm{pm} / \mathrm{mT}$ and $0.950 \mathrm{pm} / \mathrm{mT}$, respectively. $\mathrm{TbDyFe} / \mathrm{FeNi}$ multilayer has a sensitivity response of $1.08 \mathrm{pm} / \mathrm{mT}$, which is already two times higher than that of the $1-\mu \mathrm{m}$ TbDyFe single layer. The magnetic field response is linear and reversible. By structure and multilayer design, the proposed work not only can miniaturize sensor size (thin film instead of bulk material), but also can improve the response sensitivity to the magnetic field, which is very promising for magnetic sensor application.

FBG sensor has been widely used in many fields due to its good electric insulation, small volume and high sensitivity. Therefore utilizing FBG to monitor electric current is very meaningful. Current can be 
measured by detecting magnetic field around the current cable. At present most researches are based on bulk magnetostrictive materials [36-38] and magneto-optical crystal [39-41], and there are rarely investigations using FBG dipped in the magnetic fluid as sensing element. It was reported that long-period fiber grating (LPFG) had a high wavelength shift [42] when immersed in the magnetic fluid. However, LPFG is not easy for commercial application due to its broad full wavelength at half maximum (FWHM) which limits the measurement accuracy. It is also demonstrated that the magnetic fluid can be used to detect magnetic field by Faraday effect [43], but this method is susceptible to the effect of the light source's intensity. As a new alternative solution, FBG combined magnetic fluid as magnetic sensitive probe was proposed.

As is well known, the reflected wavelength $\left(\lambda_{B}\right)$ of the FBG has correlation with its effective refractive index $\left(n_{\text {eff }}\right)$ and grating pitch ( 1$)$. The equation can be expressed as [44]:

$$
\lambda_{B}=2 n_{\text {eff }} \Lambda \text {. }
$$

In general conditions, the $n_{\text {eff }}$ of the FBG is not influenced by the external refractive index. However, if fiber cladding diameter is reduced along the grating region to a certain extent, the $n_{\text {eff }}$ is significantly affected by external refractive index [45]. FBG refractive index sensors are based on the interactions between the evanescent field of the fundamental core mode and the surrounding materials.

Magnetic fluid is a kind of stable colloidal solution of ferromagnetic nanoparticles. The behaviors of ferromagnetic particles in the magnetic fluid are dependent on the external magnetic field, so the refractive index of the magnetic fluid is shown to be magnetic field dependent [46-48]. The refractive index $n$ is expressed in the following equation:

$$
n=\sqrt{\varepsilon_{r}}=\sqrt{1+\chi}
$$

where $\varepsilon_{r}$ represents the dielectric constant, and $\chi$ is the electric susceptibility. When the external magnetic field is perpendicular to the propagation direction of light, we have

$$
\frac{\partial \chi}{\partial H}<0
$$

So the magnetic fluid's refractive index will decrease when the magnetic field increases. The principle of our research relies on the dependence of the Bragg resonance on the effective refractive index $n_{\text {eff }}$ caused by the magnetic fluid setting in changing magnetic field. So the $\lambda_{B}$ of the FBG has correspondence with the magnetic field value. By utilizing the magneto-optical tenability of the magnetic fluid' refractive index, we have proposed a cladding-etched FBG with the magnetic fluid as magnetic sensor.

Nanosized $\mathrm{Fe}_{3} \mathrm{O}_{4}$ magnetic fluid were prepared by the chemical co-precipitation method [49]. The ion reaction equation is described in the following form:

$$
\mathrm{Fe}^{2+}+\mathrm{Fe}^{3+}+8 \mathrm{OH}^{-}=\mathrm{Fe}_{3} \mathrm{O}_{4}+4 \mathrm{H}_{2} \mathrm{O} \text {. }
$$

In the synthesis process, $\mathrm{Fe}^{2+}$ was added in excess ratio to get stable $\mathrm{Fe}_{3} \mathrm{O}_{4}$ magnetic fluid with the super paramagnetic characteristic [50]. The molar ratio of $\mathrm{Fe}^{2+}, \mathrm{Fe}^{3+}$, and $\mathrm{OH}^{-}$is 1.2:1:8. Trisodium citrate dihydrate was mixed in the solution as surfactant. The mixed solution was stirred in a flask for 2 hours at $85^{\circ} \mathrm{C}$. Then solution was moved to a beaker. After de-ioned water's washing and ultrasonic vibrating, a stable solution was obtained. The solution was diluted with de-ioned water to get a refractive index near the cladding of the FBG. In our experiment, the quality of the $\mathrm{Fe}_{3} \mathrm{O}_{4}$ in solution was approximately $2 \%$.

The $\lambda_{B}$ of the FBG is $1307.857 \mathrm{~nm}$ at room temperature of $25^{\circ} \mathrm{C}$. Single mode FBG fiber was dipped into mixed HF solvent to remove cladding layer at room temperature. $40 \%$ hydrofluoric acid solution was diluted by ethanol and de-ioned water with 1:1:1 in volume. FBG's diameter was reduced by controlling the etching time. To get the desired diameter of the FBG, single mode fiber etched at the same condition was measured with optical 
microscopy during the etching process. After the etching process, the magnetic fluid was slowly injected into the micro-tube for magnetic field measurement. Figure 11 shows different etched FBGs enlarged by 1000 times in length. Obtained by optical microscopy, the diameters of the fiber are $11.32 \mu \mathrm{m}, 9.98 \mu \mathrm{m}, 8.53 \mu \mathrm{m}$, respectively.

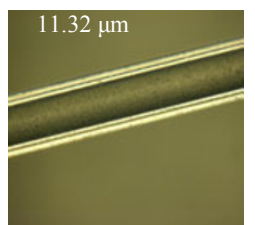

(a)

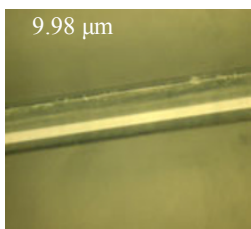

(b)

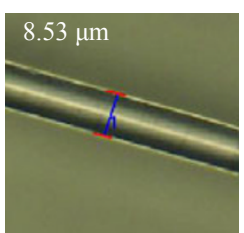

(c)
Fig. 11 Microphotography of the chemically-etched FBG with different diameters.

Commercially, available software RSoft was used to simulate the wavelength response of the etched FBG under different external refractive indices in the simulation. The central wavelength shift of the etched FBG was simulated by the coupled-mode theory [51] under a three-layer step index fiber geometry. During the simulation, the diameter of the fiber core was set as $8.4 \mu \mathrm{m}$. The refractive indices of the cladding and core of the fiber were set as 1.32 and 1.47 , respectively. The $\lambda_{B}$ of the FBG with different thickness claddings were calculated by changing the refractive index of the background. From Fig. 12, It can be concluded that the center wavelength of the etched FBG shifts to longer wavelength with an increase of the ambient refractive index. The resonant wavelength of the etched FBG shifts sharply when the ambient refractive index is close to the cladding refractive index of the etched FBG, i.e., near 1.460. The etched FBG with small diameter has higher sensitivity at the same ambient refractive index.

Figure 13 is the schematic diagram of the fiber-magnetic sensor. During magnetic field characterization, the reflected wavelength is collected with a BCD-100 FBG demodulator. An FFP-TF from Micron Optics Inc., USA is installed in the demodulator as core component for wavelength demodulation. The resolution of the demodulator is $1 \mathrm{pm}$. The varying magnetic field is provided by changing two permanent magnets' distance, and a Lake Shore 460 Gauss meter is employed to calibrate the magnetic intensity.

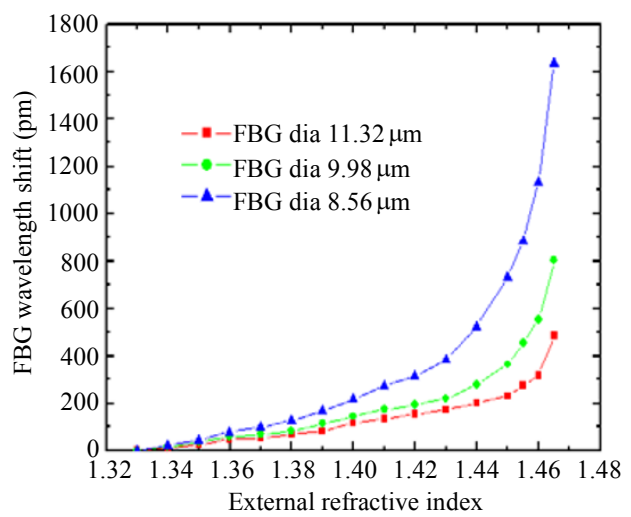

Fig. 12 Theoretical simulation of the etched FBG response to different ambient refractive indices.

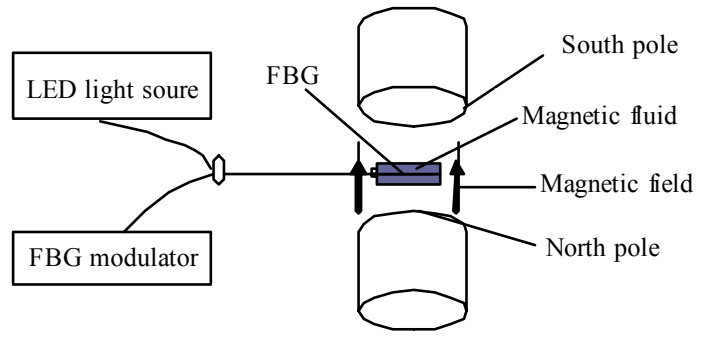

Fig. 13 Schematic diagram of the fiber-magnetic sensor.

Figure 14 shows the shift of the FBG's wavelength during an increase of the magnetic field from 0 to $24 \mathrm{mT}$. As a reference, a standard FBG with a diameter of $125 \mu \mathrm{m}$ is not etched by the HF solvent, and it has no wavelength shift when the magnetic field changes. As the magnetic field increases, the reflected wavelength of etched FBGs with different diameters shifts to shorter wavelength. The wavelength shift of the etched FBG shows a nonlinear dependence on the magnetic field, which is similar to the theoretical simulation of the etched FBG under different ambient refractive indices. When the magnetic field increases to $24 \mathrm{mT}$, the wavelength shifts of the FBG with diameters of $11.32 \mu \mathrm{m}, 9.98 \mu \mathrm{m}$ and $8.53 \mu \mathrm{m}$ are $25 \mathrm{pm}, 46 \mathrm{pm}$ and $86 \mathrm{pm}$, respectively. The response of the sensor is about 15 seconds. It can be also concluded from Fig. 4 that the FBG with smaller diameter has larger 
wavelength shift in the magnetic field.

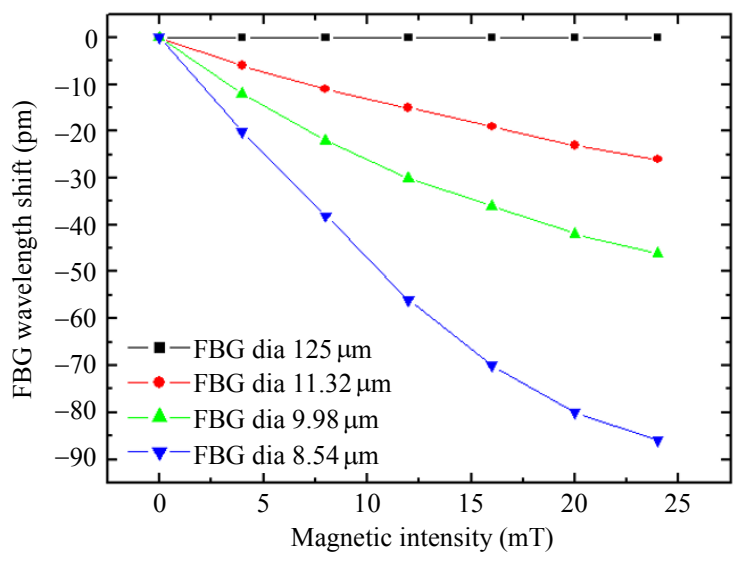

Fig. 14 Central wavelength shift of different etched FBGs dipped in magnetic fluid under different magnetic intensities.

The reason for this result is that when there is no magnetic field, the ferromagnetic particles are uniformly dispersed in the solvent. The refractive index magnetic fluid is the highest one without the magnetic field. When there is magnetic field, ferromagnetic particles will be magnetized to form lager ferromagnetic particles and magnetic chains along the direction of the magnetic field. The arrangement of nanoparticles has close relationship with the external magnetic field's intensity, leading to a decreased refractive index of the magnetic fluid with an increased magnetic field. This phenomenon is explained by the magneto-electric effect [52] of the magnetic fluid. So the reflected wavelength of the FBG shifts to shorter wavelength with the effective refractive index changed by magnetic fluid setting in an increased magnetic field. In experiment, the drift of the etched FBG is not as big as that being simulated. The reason for this phenomenon is mainly due to relatively small change of the magnetic fluid's refractive index.

Figure 15 illustrates the sensor's repeatability in three cycles. The maximum magnetic field is $16 \mathrm{mT}$ in this process. FBGs with different diameters of $11.32 \mu \mathrm{m}, 9.98 \mu \mathrm{m}$ and $8.54 \mu \mathrm{m}$ show good reversibility at the maximum wavelength shift of $18 \mathrm{pm}, 36 \mathrm{pm}$ and $68 \mathrm{pm}$ respectively in the experiment. This phenomenon can be attributed to the super paramagnetic characteristic of the ferromagnetic particles. When there is no magnetic field, ferromagnetic particles keep balance due to the role of the thermal motion and the electrostatic repulsion between them. When the magnetic fluid is sated in the magnetic field, there is magnetic force between the ferromagnetic particles. After the magnetic field is removed, the ferromagnetic particles can restore to their original state by the thermal motion and electrostatic repulsion. If the magnetic field is too high, the electrostatic repulsion and thermal motion can not eliminate the adhesion force between ferromagnetic particles and magnetic chains. So the refractive index of the magnetic fluid will be permanently changed. This is the main reason that the sensor's reversibility is not good when the magnetic field is more than $16 \mathrm{mT}$.

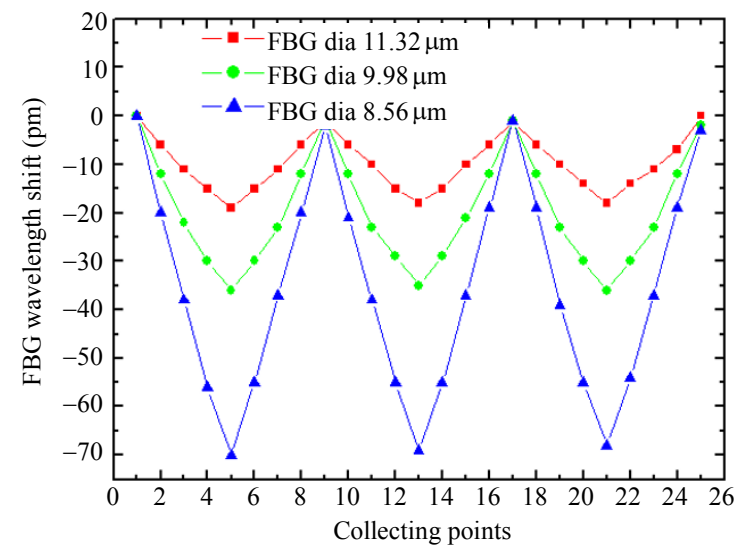

Fig. 15 Three cycles of the magnetic response of the FBG with diameters of $11.32 \mu \mathrm{m}, 9.98 \mu \mathrm{m}$ and $8.56 \mu \mathrm{m}$, respectively.

\section{Conclusions and remarks}

The combination of fiber optics with the nano-structure technology and sensitive thin film offers great potential for the realization of novel sensor concepts. Miniatured optical fiber sensors with thin films as sensitive elements could open new fields for optical fiber sensor applications. Thin films work as sensitive elements and transducer to get response and feedback from environments, and optical fiber here is employed to work as signal carrier. This paper presents some research work 
conducted at the National Engineering Laboratory for Optical Fiber Sensors in recent years. $\mathrm{Pd} / \mathrm{WO}_{3}$ co-sputtered coating as sensing material for optical hydrogen sensors shows robust mechanical stability and meanwhile good sensing performance. TbDyFe magnetostrictive coating directly deposited on an FBG demonstrates its possibility as miniature optical magnetic field/current sensors, and 40-pm shift of the FBG wavelength happens in the magnetic field in order of $50 \mathrm{mT}$. As to optical fiber magnetic sensor with the magnetic fluid as sensitive materials, the wavelength shifts of the etched FBG with diameters of $11.32 \mu \mathrm{m}, 9.98 \mu \mathrm{m}$ and $8.53 \mu \mathrm{m}$ are $25 \mathrm{pm}, 46 \mathrm{pm}$ and $86 \mathrm{pm}$ respectively when the magnetic field is $25 \mathrm{mT}$. The results show that the FBG with smaller diameter has larger sensitive wavelength shift in the magnetic field. The etched FBG shows the reverse response on the magnetic field under $16 \mathrm{mT}$, and the FBG with a diameter of $8.53 \mu \mathrm{m}$ shows good repeatability with a wavelength shift of $68 \mathrm{pm}$. As a new solution, the etched FBG combined magnetic fluid as the magnetic sensitive element could be used for magnetic field determination.

Optical fiber sensor with tin film as sensitive material could be a new area for fiber-based sensor development. The advantages are clear. Firstly, the sensor could be miniature and easy to integrate with fiber-based devices. Thin films work as sensitive elements and transducer to get response and feedback from environments, and optical fiber here are employed to work as signal carrier. Different types of sensors can be developed only with different sensitive thin films. While there are still some disadvantages, thin film can be deposited on fibers, but its mechanical stability is a key issue to be improved. Furthermore, the sensitivity of the thin film will decrease after several cycles of sensing application, which means its life is limited. However, these disadvantages could be overcome with thin film deposition technology development in the future, and therefore still exists promising prospective for thin film-based optical fiber sensors.

\section{Acknowledgment}

This work is finically supported by the Project of National Science Foundation of China (NSFC) (Grant No. 50830203, 60908020), Scientific Research Foundation for the Returned Overseas Chinese Scholars, State Education Ministry, and Program for New Century Excellent Talents in University (NCET-10-0664).

Open Access This article is distributed under the terms of the Creative Commons Attribution License which permits any use, distribution, and reproduction in any medium, provided the original author(s) and source are credited.

\section{References}

[1] M. Tabib-Azar, B. Sutapun, R. Petrick, and A. Kazemi, "Highly sensitive hydrogen sensors using palladium coated fiber optics with exposed cores and evanescent field interactions," Sensors and Actuators B: Chemical, vol. 56, no. 1-3, pp. 158-163, 1999.

[2] S. K. Khijwania and B. D. Gupta, "Fiber optic evanescent field absorption sensor: effect of fiber parameters and geometry of the probe," Optical and Quantum Electronics, vol. 31, no. 8, pp. 625-636, 1999.

[3] S. Sekimoto, H. Nakagawa, S. Okazaki, K. Fukuda, S. Asakura, T. Shigemori, and S. Takahashi, "A fiber-optic evanescent-wave hydrogen gas sensor using palladium-supported tungsten oxide," Sensors and Actuators B: Chemical, vol. 66, no. 1, pp. 142-145, 2000.

[4] J. Villatoro and D. Monzón-Hernándea, "Fast detection of hydrogen with nano fiber tapers coated with ultra thin palladium layers," Optics Express, vol. 13, no. 13, pp. 5087-5092, 2005.

[5] D. Monzón-Hernández, D. Luna-Moreno, and D. Martínez-Escobar, "Fast response fiber optic hydrogen sensor based on palladium and gold nano-layers," Sensors and Actuators B: Chemical, vol. 136, no. 2, pp. 562-566, 2009.

[6] M. Yang, Y. Sun, D. Zhang, and D. Jiang, "Using $\mathrm{Pd} / \mathrm{WO}_{3}$ composite thin films as sensing materials for optical fiber hydrogen sensors," Sensors and 
Actuators B: Chemical, vol. 143, no. 2, pp. 750-753, 2010.

[7] M. A. Butler, "Micromirror optical-fiber hydrogen sensor," Sensors and Actuators B: Chemical, vol. 22, no. 2, pp. 155-163, 1994.

[8] X. B évenot, A. Trouillet, C. Veillas, H. Gagnaire, and M. Clément, "Hydrogen leak detection using an optical fiber sensor for aerospace applications," Sensors and Actuators B: Chemical, vol. 67, no. 1-2, pp. 57-67, 2000.

[9] K. Lin, Y. Lu, J. Chen, R. Zheng, P. Wang, and H. Ming, "Surface plasmon resonance hydrogen sensor based on metallic grating with high sensitivity," Optics Express, vol. 16, no. 23, pp. 18599-18604, 2008.

[10] C. Ma and A. Wang, "Optical fiber tip acoustic resonator for hydrogen sensing," Optics Letters, vol. 35, no. 12, pp. 2043-2045, 2010.

[11] M. Buric, T. Chen, M. Maklad, P. R. Swinehart, and K. P. Chen, "Multiplexable low-temperature fiber Bragg grating hydrogen sensors," IEEE Photonics Technology Letters, vol. 21, no. 21, pp. 1594-1596, 2009.

[12] B. Sutapun, M. Tabib-Azar, and A. Kazemi, "Pd-coated elastooptic fiber optic Bragg grating sensors for multiplexed hydrogen sensing," Sensors and Actuators B: Chemical, vol. 60, no. 1, pp. 27-34, 1999.

[13] C. Caucheteur, M. Debliquy, D. Lahem, and P. Mégret, "Hybrid fiber gratings coated with a catalytic sensitive layer for hydrogen sensing in air," Optics Express, vol. 20, no. 21, pp. 16854-16859, 2008.

[14] M. Aleixandrea, P. Correderab, M. L. Hernanzb, et al., "Study of a palladium coated Bragg grating sensor to detect and measure low hydrogen concentrations," in Proc. 2007 Spanish Conference on electron devices (IEEE 4271210), Madrid, Jan. 31-Feb. 2, pp. 223-225, 2007.

[15] J. H. Lee, J. H. Kim, Y. G. Han, S. H. Kim, and S. B. Lee, "Investigation of Raman fiber laser temperature probe based on fiber Bragg gratings for long-distance remote sensing applications," Optics Express, vol. 12, no. 8, pp. 1747-1752, 2004.

[16] G. T. Kanellos, G. Papaioannou, D. Tsiokos, C. Mitrogiannics, G. Nianios, and N. Pleros, "Two dimensional polymer-embedded quasidistributed FBG pressure sensor for biomedical applications," Optics Express, vol. 18, no. 1, pp. 179-186, 2010.

[17] D. Lin, X. Jiang, F. Xie, W. Zhang, Z. Lin, and I. Bennion, "High stability multiplexed fiber interferometer and its application on absolute displacement measurement and on-line surface metrology," Optics Express, vol. 12, no. 23, pp. 5729-5734, 2004.

[18] J. Zhou, X. Dong, and Z. Shi, "Theoretical and experimental investigation of bending sensitivity of the D-shaped fiber Bragg gratings," Chinese Acta Photonica Sinica, vol. 35, no. 11, pp. 1734-1737, 2006 (in Chinese).

[19] C. Tien, H. Chen, W. Liu, S. Jyu, S. Lin, and Y. Lin, "Hydrogen sensor based on side-polished fiber Bragg gratings coated with thin palladium film," Thin Solid Films, vol. 516, no. 16, pp. 5360-5363, 2008.

[20] D. Kersey, M. A. Davis, H. J. Patrick, M. LeBlanc, K. P. Koo, C. G. Askins, M. A. Putnam, and E. J. Friebele, "Fiber grating sensors," Journal of Lightwave Technology, vol. 15, no. 8, pp. 1442-1463, 1997.

[21] K. O. Hill, B. Malo, F. Bilodeau, D, C. Johnson, and J. Albert, "Bragg gratings using fabricated in monomode photosensitive optical fiber by UV exposure thorough a phase mask," Apply Physics Letter, vol. 62, no. 10, pp. 1035-1037, 1993.

[22] Z. Chen and L. Liu, "Wavelength tuning of fiber Bragg grating based on fiber side polishing," in Proc. SPIE, vol. 7157, pp. 71570J-1-71570J-6, 2009.

[23] A. Yariv and H. V. Winsor, "Proposal for detection of magnetic fields through magnetostrictive perturbation of optical fibers," Optics Letters, vol. 5, no. 3, pp. 87-89, 1980.

[24] S. C. Rashleigh, "Magnetic-field sensing with a single-mode fiber," Optics Letters, vol. 6, no. 1, pp. 19-21, 1981.

[25] A. D. Kersey, D. A. Jackson, and M. Corke, "Single-mode fiber optic magnetometer with DC bias field stabilization," Journal of Lightwave Technology, vol. 3, no. 4. pp. 836-840, 1985.

[26] J. Jarzynski, J. H. Cole, J. A. Bucaro, and C. M. Davis, "Magnetic field sensitivity of an optical fiber with magnetostrictive jacket," Applied Optics, vol. 19, no. 22, pp. 3746-3748, 1980.

[27] F. Bucholtz, Fiber optic sensors: an introduction for engineers and scientists, E. Udd Ed. New York: Wiley, 1991.

[28] M. Sedlar and L. Pust, "Preparation of cobalt doped nickel ferrite thin films on optical fibers by dip-coating technique," Ceramics International, vol. 21, no. 1, pp. 21-27, 1995.

[29] G. Vértesy, A. Gasparics, and Z. Vértesy, "Improving the sensitivity of Fluxset magnetometer by processing of the sensor core," Journal of Magnetism and Magnetic Materials, vol. 196-197, no. 1, pp. 333-334, 1999.

[30] M. Sedlar, "The preparation and magnetic properties of sodium modified iron oxide thin films by a sol-gel method," Ceramics Internetional, vol. 20, no. 1, pp. 73-78, 1994.

[31] J. Mora, A. Diez, J. L. Cruz, and M. V. Andrs, "A magnetostrictive sensor interrogated by fiber gratings for DC-current and temperature discrimination," IEEE Photonics Technology Letters, vol. 12, no. 12, pp. 1680-1682, 2000. 
[32] W. Liang, Y. Huang, Y. Xu, R. K. Lee, and A. Yariv, "Highly sensitive fiber Bragg grating refractive index sensors," Applied Physics Letters, vol. 86, no. 15, pp. 151122, 2005.

[33] J. A. Garcia-Souto and H. Lamela-Rivera, "High resolution $(<1 \mathrm{~nm})$ interferometric fiber-optic sensor of vibrations in high-power transformers," Optics Express, vol. 14, no. 21, pp. 9679-9686, 2006.

[34] N. Tiercelin, V. Preobrazhensky, P. Pernod, and A. Ostaschenko, "Enhanced magnetoelectric effect in nanostructured magnetostrictive thin film resonant actuator with field induced spin reorientation transition," Applied Physics Letters, vol. 92, no. 6, pp. 062904, 2008.

[35] C. Shi, J. Chen, G. Wu, X. Li, J. Zhou, and F. Ou, "Stable dynamic detection scheme for magnetostrictive fiber-optic interferometric sensors," Optics Express, vol. 14, no. 12, pp. 5098-5102, 2006.

[36] K. Chiang, R. Kancheti, and V. Rastogi, "Temperature-compensated fiber-Bragg-grating-based magnetostrictive sensor for $\mathrm{dc}$ and ac currents," Optical Engineering, vol. 42, no. 7, pp. 1906-1909, 2003.

[37] B. Yi, B. Chu, and K. Chiang, "Temperature compensation for fiber-Bragg-grating-based magnetostrictive sensor," Microwave and Optical Technology Letters, vol. 36, no. 3, pp. 211-213, 2003.

[38] Y. Liu, K. S. Chiang, and P. L. Chu, "Multiplexing of temperature-compensated fiber-Bragg-grating magnetostrictive sensors with a dual-wavelength pulse laser," IEEE Photonics Technology Letters, vol. 16, no. 2, pp. 572-574, 2004.

[39] N. Itoh, H. Minemoto, D. Ishiko, and S. Ishizuka, "Optical magnetic field sensor with high linearity using Bi-substituted rare earth iron garnet," IEEE Transaction Magnetics, vol. 31, no. 6, pp. 3191-3193, 1995.

[40] O. Kamada, T. Nakaya, and S. Higuchi, "Magnetic field optical sensors using Ce: YIG single crystals as a Faraday element," Sensors and Actuators A: Physical, vol. 119, no. 2, pp. 345-348, 2005.

[41] H. Lin and S. C. Huang, "Fiber-optics multiplexed interferometric current sensors," Sensors and Actuators A: Physical, vol. 121, no. 2, pp. 333-338, 2005.

[42] T. Liu, X. Chen, D. Yun, J. Zhang, X. Li, and J. Chen,
"Tunable magneto-optical wavelength filter of long-period fiber grating with magnetic fluids," Applied Physics Letters, vol. 91, no. 12, pp. 121116, 2007.

[43] L. Martinez, F. Cecelja, and R. Rakowski, "A novel magneto-optic magnetic fluid material for sensor applications," Sensors and Actuators A: Physical, vol. 123-124, pp. 438-443, 2005.

[44] A. D. Kersey, M. A. Davis, H. J. Patrick, M. LeBlanc, K. P. Koo, C. G. Askins, M. A. Putnam, and E. J. Friebele, "Fiber grating sensors," Journal of Lightwave Technology, vol. 15, no. 8, pp. 1442-1463, 1997.

[45] A. Iadicicco, A. Cusano H. Kumazaki, A. Cutolo, R. Bernini, and M. Giordano, "Thinned fiber Bragg gratings as high sensitivity refractive index sensor," IEEE Photonics Technology Letters, vol. 16, no. 4, pp. 1149-1151, 2004.

[46] S. Y. Yang, Y. F. Chen, H. E. Horng, C. Y. Hong, W. S. Tse, and H. C. Yang, "Magnetically-modulated refractive index of magnetic fluid films," Applied Physics Letters, vol. 81, no. 26, pp. 4931-4933, 2002.

[47] H. E. Horng, C. Y. Hong, S. Y. Yang, and H. C. Yang, "Designing the refractive indices by using maganetic fluids," Applide Physics Letters, vol. 15, no. 15, pp. 2434-2436, 2003.

[48] C. Y. Hong, S. Y. Yang, H. E. Horng, and H. C. Yang, "Control parameters for the tunable refractive index of magnetic fluid films," Joural Applied Physics, vol. 94, no. 6, pp. 3849-3852, 2003.

[49] Y. Laiqiong, Z. Luji, and Y. Jixiao, "Study of preparation and properties on magnetization and stability for ferromagnetic fluids," Materials Chemistry and Physics, vol. 66, no. 1, pp. 6-9, 2000.

[50] J. Huang, R. Zhao, H. Wang, W. Zhao, and L. Ding, "Immobilization of glucose oxidase on $\mathrm{Fe}_{3} \mathrm{O}_{4} / \mathrm{SiO}_{2}$ magnetic nanoparticles," Biotechnol Letters, vol. 32, no. 6, pp. 817-821, 2010.

[51] S. S. Orlow, A. Yariv, and S. V. Essen, "Coupled-mode analysis of fiber-optic add-drop filters for dense wavelength-division multiplexing," Optics letters, vol. 22, no. 10, pp. 688-690, 1991.

[52] A. J. Mailfert and B. Nahounou, "Dielectric behavior of a magnetic fluid subjected to a uniform magnetic field," IEEE Transactions on Magnetics, vol. 16, no. 2, pp. 254-257, 1980. 